\title{
Slow Wave Phenomena in Photonic Crystals
}

\author{
Alex Figotin and Ilya Vitebskiy
}

\begin{abstract}
Slow light in photonic crystals and other periodic structures is associated with stationary points of the photonic dispersion relation, where the group velocity of light vanishes. We show that in certain cases, the vanishing group velocity is accompanied by the so-called frozen mode regime, when the incident light can be completely converted into the slow mode with huge diverging amplitude. The frozen mode regime is a qualitatively new wave phenomenon it does not reduce to any known electromagnetic resonance. Formally, the frozen mode regime is not a resonance, in a sense that it is not particularly sensitive to the size and shape of the photonic crystal. The frozen mode regime is more robust and powerful, compared to any known slow-wave resonance. It has much higher tolerance to absorption and structural imperfections.
\end{abstract}

PACS: 42.25.-p, 42.70.-a, 42.70.Qs 


\section{Introduction}

\subsection{What is slow light?}

The velocity of light in transparent dispersive media is characterized by two different physical quantities - the phase velocity and the group velocity. The phase velocity of a travelling wave is defined as

$$
v_{p h}=\omega / k,
$$

where $\omega$ is the frequency and $k$ is the wave number. The group velocity of a travelling wave is defined as

$$
v_{g}=\partial \omega / \partial k .
$$

The group velocity of light is one of the most important electromagnetic characteristics of transparent media. It determines the speed of pulse propagation and is usually referred to simply as the speed of light propagation. With certain reservations, the group velocity also coincides with electromagnetic energy velocity in a wave packet [1, 2]. The definitions (1) and (2) apply not only to uniform transparent media and waveguides, but also to nonuniform spatially periodic structures, such as photonic crystals, arrays of coupled optical resonators, etc. In the case of a nonuniform periodic structure, $k$ is the Bloch wave number defined in the Brillouin zone [1]. In a slow light case, the electromagnetic pulse propagates through the dispersive medium with the speed $v_{g} \ll c$, while the respective value of the phase velocity (11) is irrelevant.

The term "slow light" has been widely used to describe a broad range of qualitatively different physical phenomena. There have been hundreds of publications in which the above term appears as a key word, while in many cases, the physical phenomena in question have little in common. Below, we just briefly outline the most important situations in which the concept of slow light can be invoked, and then we turn to the main subject of this paper - the frozen mode regime.

Most of the "slow light" cases can be grouped into two major categories:

- those where the low group velocity of light results from strong temporal (frequency) dispersion of the transparent medium;

- those where the low speed of pulse propagation is a result of coherent interference in spatially periodic structures.

\subsection{Slow light in media with strong temporal dispersion}

In a uniform medium, both the phase and the group velocities of light can be expressed in terms of the refractive index $n$

$$
\begin{gathered}
v_{f}=c / n, \\
v_{g}=c\left(n+\omega \frac{d n}{d \omega}\right)^{-1} .
\end{gathered}
$$

The derivative $d n / d \omega$ in (4) characterizes the frequency dispersion of the medium. At optical frequencies, the refractive index $n$ of common transparent substances does not exceed several units, and the speed of light propagation is of the same order of magnitude as in vacuum. The situation can change dramatically in a strongly dispersive medium where the term $\omega d n / d \omega$ in (4) becomes dominant

$$
\text { if } \omega \frac{d n}{d \omega} \gg n \text {, then } v_{g} \approx c\left(\omega \frac{d n}{d \omega}\right)^{-1} \ll c \text {. }
$$


Strong frequency dependence of the refractive index $n$ implies that the light group velocity can be substantially different from $c$. The relation (5) yields the following limitation on the slow pulse bandwidth

$$
\frac{\Delta \omega}{\omega} \ll \frac{v_{g}}{c},
$$

where the assumption is made that the refractive index $n$ within the transparency window is of the order of unity. The condition (6) can also be viewed as a constraint on the minimal propagation speed of a pulse with a given bandwidth $\Delta \omega$.

Usually, the large temporal dispersion (5) is a result of excitation of electronic or some other intrinsic resonances of the medium. This always involves some kind of a delicate resonant light-matter interaction with extremely small bandwidth. For instance, in the well known case of electromagnetically induced transparency [3, 4, 5, 6, 7, 8, 9, 10, the incident light interacts with atomic spin excitations forming the so-called dark-state polaritons. These polaritons propagate slowly through the medium in the form of a sharply compressed pulse, the energy of which is much smaller than that of the incident light pulse. In another example, the slow wave is associated with plasmons, which are electron density waves in gas of mobile electrons of the metal. Plasmons can also interact with light forming polaritons. There are many more examples of condensed matter excitations, strongly interacting with light and having low group velocity and relatively low relaxation rate. In most of such cases, the slow pulse propagating through the dispersive medium can be seen as an intrinsic coherent excitation (a polariton) triggered by the input light, rather than a light pulse per se. The group velocity of such an excitation may have little to do with the Maxwell equations and the speed of light $c$.

In the rest of the paper we focus exclusively on the cases not involving any intrinsic excitations of the medium and, therefore, not related to temporal dispersion. Instead, we will focus on spatially periodic structures, in which low speed of electromagnetic pulse propagation results solely from spatial inhomogeniety.

\subsection{Slow light in spatially periodic structures}

Well-known examples of periodic dielectric structures include photonic crystals 11, 12, 13, 14, periodic arrays of coupled optical resonators [15, 16, 17, 18, 19, 20, 21, 22, 23, and photonic crystal coupled waveguide [24, 25, 26, 27, 28, 29, 30, 31. Low speed of pulse propagation in all these cases is the result of coherent interference of light scattered at the interfaces of adjacent structural components. The effects of spatial dispersion associated with structural periodicity are particularly strong when the structural period $L$ and the light wavelength $\lambda$ are comparable in value

$$
L \sim \lambda .
$$

Strong spatial dispersion can result in low or even zero group velocity of the respective Bloch wave. In practice, at optical frequencies, the speed of pulse propagation in periodic dielectric arrays can be reduced by not more than three orders of magnitude. This is not a fundamental restriction, but rather a technological limitation related to the difficulty of building flawless periodic arrays at nanoscales. On the positive side, the dielectric components of the periodic array are not required to display strong temporal dispersion. As a consequence, the absorption of light is not an inherent problem in this case. 
There is a natural bandwidth limitation on the slow pulse in periodic dielectric arrays, which is similar to the case of slow light in time-dispersive media. Let $\Delta \omega$ be the frequency bandwidth of a pulse and $\Delta k$ - the respective range of the Bloch wave number. The average group velocity $\left\langle v_{g}\right\rangle$ of the pulse can be defined as

$$
\left\langle v_{g}\right\rangle \approx \frac{\Delta \omega}{\Delta k} .
$$

Let us assume that the pulse propagating in the periodic medium is composed of the Bloch eigenmodes belonging to the same spectral branch of the dispersion relation $\omega(k)$. This assumption implies that $\Delta k$ cannot exceed the size $2 \pi / L$ of the Brillouin zone

$$
\Delta k<2 \pi / L,
$$

where $L$ is the unit cell length of the periodic array. In addition, we assume that the refractive index of the constitutive components of the periodic array is of the order of unity and, therefore,

$$
L \sim \lambda_{0}=2 \pi c / \omega
$$

where $\lambda_{0}$ is the light wavelength in vacuum. The relations (8) and (9) yield the following limitation on the minimal propagation speed of a pulse with a given bandwidth $\Delta \omega$

$$
\left\langle v_{g}\right\rangle>\frac{L}{2 \pi} \Delta \omega \sim c \frac{\Delta \omega}{\omega} .
$$

The restriction (10) is similar to that defined by the inequality (6) and related to the case of slow light in a uniform medium with temporal (frequency) dispersion.

\subsection{Examples of periodic arrays supporting slow light}

During the last two decades, a tremendous progress has been made in theory and applications of periodic arrays of coupled optical resonators. Generally, if the coupling between adjacent resonators in a periodic array is weak, the group velocity of Bloch excitations propagating through the array is very low in each and every transmission band. This is true regardless of the nature of individual resonators. The above simple idea forms the basis for one of the most popular approaches to slowing down the light. One inevitable consequence of the weak coupling between the neighboring optical resonators is that all the individual transmission bands are very narrow. Another problem is that there can be severe restrictions on transmitted power. An extensive discussion on the subject and numerous examples and references can be found in [15, 16, 17, 18, 19, 20, 21, 22, 23. A qualitatively similar situation occurs in linedefect waveguides in photonic crystals, where a periodic array of structural defects plays the role of weakly coupled optical resonators [24, 25, 26, 27, 28, 29, 30, 31.

Slow light phenomena in periodic arrays of weakly coupled resonators have been the subject of a great number of recent publications, including some excellent review articles cited above. A common characteristic of all different realizations of this approach is a relatively low density of modes. One consequence of this is a significant nonlinearity. In certain cases, the nonlinearity is so extreme that it occurs on a single photon level. Strong nonlinearity can be useful in controlling the flow of light. On the other hand, it can severely limit the transmission capabilities of the optical waveguide. In this respect, photonic crystals have advantage. 
Photonic crystals are spatially periodic structures composed of two or more different transparent dielectric materials 11, 12, 13, 14. Unlike the case of optical waveguides and linear arrays of coupled resonators, in photonic crystals we have bulk electromagnetic waves capable of propagating in any direction through the periodic heterogeneous structure. This results in much greater density of modes, compared to arrays of weakly coupled resonators [32, 33, 34].

Strong spatial dispersion of a typical photonic crystal and any other periodic dielectric structure is reflected in a complicated $k-\omega$ diagram featuring transmission bands and gaps. Normally, each spectral branch $\omega(k)$ of the Bloch dispersion relation develops stationary points $\omega_{s}=\omega\left(k_{s}\right)$ where the group velocity (2) of the corresponding propagating mode vanishes

$$
d \omega / d k=0, \text { at } \omega=\omega_{s}=\omega\left(k_{s}\right) .
$$

Examples of different stationary points are shown in Fig. 1. Each of the frequencies $\omega_{g}$, $\omega_{0}$ and $\omega_{d}$ is associated with zero group velocity of the respective traveling wave, but there are some fundamental differences between those three cases. These differences are particularly pronounced when it comes to the efficiency of conversion of the incident light into the slow mode inside the periodic structure. In most cases, incident radiation with the frequency of one of the slow modes is simply reflected back to space, without exciting the slow mode inside the periodic structure. How to overcome this fundamental problem and, thereby, how to transform a significant fraction of the incident light energy into a slow mode with drastically enhanced amplitude, is the primary subject of this paper.

\section{Frozen mode regime in photonic crystals.}

The effects of strong spatial dispersion in periodic dielectric structures culminate at stationary points (11) of the Bloch dispersion relation, where the group velocity (2) of a traveling Bloch wave vanishes. One reason for this is that vanishing group velocity always implies a dramatic increase in density of modes at the respective frequency. In addition, vanishing group velocity also implies certain qualitative changes in the eigenmode structure, which can strongly affect the propagation and scattering of light. A particular example of the kind is the frozen mode regime associated with a dramatic enhancement of the amplitude of the wave transmitted to the periodic medium [35, 36, 37, 38. There are at least two qualitatively different modifications of the frozen mode regime, each related to a specific singularity of the electromagnetic dispersion relation. Both effects can be explained using a simple example of a plane electromagnetic wave normally incident on a lossless semi-infinite periodic structure.

The frozen mode regime of the first kind is associated with a stationary inflection point (SIP) of the $k-\omega$ diagram shown in Fig. 1(b). In the vicinity of stationary inflection point, the relation between the frequency $\omega$ and the Bloch wave number $k$ can be approximated as

$$
\omega-\omega_{0} \propto\left(k-k_{0}\right)^{3} .
$$

A monochromatic plane wave of frequency close to $\omega_{0}$ incident on semi-infinite photonic crystal is converted into the frozen mode with infinitesimal group velocity and dramatically enhanced amplitude, as illustrated in Fig. 2. The saturation value of the frozen mode amplitude diverges as the frequency approaches the SIP value $\omega_{0}$, as shown in Fig. 2(c). Remarkably, the photonic crystal reflectivity at $\omega=\omega_{0}$ can be very 
low, implying that the incident radiation is almost totally converted into the frozen mode with zero group velocity, drastically enhanced amplitude, and finite energy flux close to that of the incident wave. This remarkable phenomenon is uniquely associated with a stationary inflection point (SIP) of the Bloch dispersion relation. Not every periodic structure can display such a spectral singularity, but if it does, one can expect the behavior described in Fig. 2 .

A qualitatively different kind of frozen mode regime can occur in the vicinity of a degenerate photonic band edge (DBE) shown in Fig. 1(c). At frequencies just below $\omega_{d}$, the respective dispersion relation can be approximated as

$$
\omega_{d}-\omega \propto\left(k-k_{d}\right)^{4}, \text { at } \omega \lesssim \omega_{d} .
$$

Contrary to the case of stationary inflection point (12), in the vicinity of a degenerate band edge the photonic crystal becomes totally reflective. But at the same time, the steady-state field inside the periodic medium (at $z>0$ ) develops a very large amplitude, diverging as the frequency approaches its critical value $\omega_{d}$. Such a phenomenon is illustrated in Fig. 3. The frozen mode profile below and above the degenerate band edge frequency $\omega_{d}$ is different. It has a large saturation value at frequencies located inside the transmission band (at $\omega \leq \omega_{d}$ ), as seen in Fig. B(a) and (b). This saturation value diverges as $\omega \rightarrow \omega_{d}-0$. By contrast, at frequencies lying inside the band gap (at $\omega>\omega_{d}$ ), the field amplitude initially increases dramatically with the distance $z$ from the surface, but then vanishes as the distance $z$ further increases, as seen in Fig. $3(\mathrm{~d}-\mathrm{f})$.

Hereinafter, the above two phenomena will be referred to as the SIP-related frozen mode regime and the DBE-related frozen mode regime, respectively. Figs. 2 and 3 describe the frozen mode regime in hypothetical lossless semi-infinite photonic crystal. What happens to the frozen mode regime in a plane-parallel photonic slab with a finite thickness $D$ ?

First, let us assume that the incident light frequency is equal to that of the respective stationary point $\left(\omega_{0}\right.$ or $\left.\omega_{d}\right)$ of the Bloch dispersion relation in Fig. 1 In either case, in the leftmost portion of the photonic slab, the frozen mode profile remains the same as in the semi-infinite case shown in Figs. 2(c) and 3(c). In the opposite, rightmost part of the photonic slab, the frozen mode amplitude now vanishes, as shown in Fig. $4(\mathrm{a}-\mathrm{c})$. In either case, the maximum field intensity $W_{\max }$ inside the photonic slab is

$$
W_{\max } \propto W_{I} N^{2},
$$

where $W_{I}$ is the intensity of the incident light and $N=D / L$ is the number of periods (primitive translations) in the $z$ direction of the finite periodic structure. Additional factors limiting the frozen mode amplitude include structural imperfections of the periodic array, absorption, nonlinearity, deviation of the incident radiation from plane monochromatic wave, etc.

Now, what happens in a finite photonic slab if the incident light frequency is slightly different from that of the respective stationary point $\omega_{0}$ or $\omega_{d}$ ?

In the case of a SIP-related frozen mode regime, the field amplitude will decrease as the frequency $\omega$ deviates from $\omega_{0}$ in either direction - similar to the case of semiinfinite structure shown in Fig. 2. In other words, in a lossless finite photonic slab, the SIP-related frozen mode regime appears as a resonance centered at $\omega_{0}$. According to (14), the light intensity at the resonance is proportional to $N^{2}$, while the respective Q-factor is proportional to $N^{3}$. 
In the case of a DBE-related frozen mode regime, the situation is quite different. Namely, in a lossless, plane-parallel photonic sample with a finite thickness, the DBErelated frozen mode regime is overwhelmed with much more powerful, giant slow wave resonance [39, 40. Let us explain this phenomenon in more detail. At DBE frequency $\omega_{d}$, the frozen mode profile in a finite photonic slab is shown in Fig. 4(a-c). According to (14), the maximum light intensity inside the photonic slab is proportional to $N^{2}$. If the incident light frequency deviates from $\omega_{d}$ toward the photonic band gap in Fig. 1(c), the light intensity inside the photonic slab will decrease, similar to the semi-infinite case shown in Figs. 33(d-f). But if the incident light frequency deviates from $\omega_{d}$ in the opposite direction (toward the transmission band in Fig. 1(c)), the light intensity inside the photonic slab will increase dramatically, peaking at a certain resonance frequency $\omega_{r}$, as shown in Fig. 5. The exact location of the resonance frequency $\omega_{r}$ is dependent on the thickness of the finite periodic structure [39, 40. But in any event, $\omega_{r}$ lies inside the transmission band and approaches the DBE frequency $\omega_{d}$ as $N$ increases. The light intensity at the frequency $\omega_{r}$ of the giant slow wave resonance is

$$
W_{\max } \propto W_{I} N^{4}
$$

which is by factor $N^{2}$ larger than that of the common slow wave resonance associated with the regular photonic band edge in Fig. 廿(a). The Q-factor of the giant slow wave resonance is proportional to $N^{5}$.

In summary, in a photonic slab of finite thickness, the SIP-related frozen mode regime persists, but its amplitude is limited by the slab thickness in accordance with the relation (14). By contrast, the DBE-related frozen mode regime in a finite planeparallel photonic slab is overrun by the giant slow wave resonance, which can be viewed as a combination of the frozen mode regime and the Fabry-Perot resonance. It is much more powerful compared to the common RBE-related slow wave resonance in a photonic slab of similar size [39, 40].

Not every periodic structure can support the frozen mode regime at normal incidence. Generally, the physical conditions for the frozen mode regime are the same as the conditions for the existence of the respective stationary points (12) or (13) of the Bloch dispersion relation. For instance, in the case of periodic layered structure, a unit cell must contain at least three layers, of which two must display a misaligned in-plane anisotropy [45, 35, 38. At optical frequencies, fabrication of such a layered structure can be challenging. Fortunately, in photonic crystals with $3 D$ periodicity, the conditions for the existence of stationary inflection point (SIP) and the related frozen mode regime are much less restrictive. A good example of the kind is provided by the inverse opal structure, where such a spectral singularity was found and investigated, both theoretically and experimentally in [46].

The frozen mode regime is a qualitatively new wave phenomenon - it does not reduce to any known electromagnetic resonance. Formally, the SIP-related frozen mode regime is not a resonance, in a sense that it is not particularly sensitive to the size and shape of the photonic crystal. Besides, the frequency dependence of the frozen mode amplitude is very different from that of a cavity resonance, or a common slow wave resonance. The frozen mode regime is much more robust, compared to any known slow-wave resonance occurring in periodic and nonperiodic photonic structures. It has much higher tolerance to absorption and structural imperfections than common Fabry-Perot or transmission band edge resonances, where the entire photonic crystal works as a resonator. 
The DBE-related frozen mode regime is quite different. In a finite plane-parallel slab it is overrun with the much more powerful giant slow-wave resonance 39, 40. The Q-factor associated with such a resonance can be by two orders of magnitude higher, compared to that of the regular slow-wave resonance in the same or similar periodic structure. More importantly, it provides the possibility of a dramatic reduction in size - up to an order of magnitude - of some basic photonic devices without compromising their performance.

\section{Physical nature of the frozen mode regime}

The essence of the frozen mode regime can be understood from the simple example of a plane monochromatic wave normally incident on a semi-infinite periodic layered structure. An important requirement, though, is that some of the layers display a misaligned in-plane anisotropy [45, 35, 38. Below we present a comparative analysis of two different kinds of frozen mode regime. Although throughout this section we only consider the case of normal incidence, most of the results and expressions remain virtually unchanged in a more general case of the frozen mode regime at oblique propagation 36, 37, 38. One difference, though, is that at oblique incidence, the frozen mode regime can occur in much simpler structures, which can have a big practical advantage.

To start with, let us introduce some basic notations and definitions. Let $\Psi_{I}, \Psi_{R}$, and $\Psi_{T}$ be the incident, reflected and transmitted waves, respectively. We assume that all three monochromatic waves propagate along the $z$ axis normal to the layers. Electromagnetic field both inside (at $z>0$ ) and outside (at $z<0$ ) the semi-infinite periodic structure is independent of the $x$ and $y$ coordinates. The transverse field components can be represented as a column-vector

$$
\Psi(z)=\left[\begin{array}{c}
E_{x}(z) \\
E_{y}(z) \\
H_{x}(z) \\
H_{y}(z)
\end{array}\right],
$$

where $\vec{E}(z)$ and $\vec{H}(z)$ are time-harmonic electric and magnetic fields. All four transverse field components in (16) are continuous functions of $z$, which leads to the following standard boundary condition at the photonic crystal interface at $z=0$

$$
\Psi_{T}(0)=\Psi_{I}(0)+\Psi_{R}(0) .
$$

Assume also that anisotropic layers of the periodic array have an in-plane birefringence

$$
\left[\begin{array}{ccc}
\varepsilon_{x x} & \varepsilon_{x y} & 0 \\
\varepsilon_{x y}^{*} & \varepsilon_{y y} & 0 \\
0 & 0 & \varepsilon_{z z}
\end{array}\right],
$$

in which case the fields $\vec{E}(z)$ and $\vec{H}(z)$ are normal to the direction of light propagation

$$
\vec{E}(z) \perp z, \vec{H}(z) \perp z .
$$

Note that the polarizations of the incident, reflected, and transmitted waves can be all different, because some of the layers of the periodic array are birefringent. The 
presence of birefringent layers is essential for the possibility of frozen mode regime. Moreover, in the case of normal incidence, each unit cell of the periodic stack must include at least two birefringent layers with misaligned anisotropy axes [45, 35, 38].

In periodic layered media, the electromagnetic eigenmodes $\Psi_{k}(z)$ are usually chosen in the Bloch form

$$
\Psi_{k}(z+L)=e^{i k L} \Psi_{k}(z),
$$

where the Bloch wave number $k$ is defined up to a multiple of $2 \pi / L$. The correspondence between $\omega$ and $k$ is referred to as the Bloch dispersion relation. Real wave numbers $k$ correspond to propagating (traveling) Bloch modes. Propagating modes belong to different spectral branches $\omega(k)$ separated by frequency gaps. The speed of a traveling wave in a periodic medium is determined by the group velocity (21). Complex wave numbers $k=k^{\prime}+i k^{\prime \prime}$ correspond to evanescent Bloch modes. Evanescent modes decay exponentially with the distance $z$ from the boundary of semi-infinite periodic structure. Therefore, under normal circumstances, evanescent contribution to the transmitted wave $\Psi_{T}(z)$ can be significant only in close proximity of the photonic crystal interface at $z=0$. The situation can change dramatically when the frequency $\omega$ approaches one of the stationary point values $\omega_{s}$. At first sight, stationary points (11) relate only to propagating Bloch modes. But in fact, in the vicinity of every stationary point frequency $\omega_{s}$, the imaginary part $k^{\prime \prime}$ of the Bloch wave number of at least one of the evanescent modes also vanishes. As a consequence, the respective evanescent mode decays very slowly, and its role may extend far beyond the photonic crystal boundary. In addition, in the special cases of interest, the electromagnetic field distribution $\Psi(z)$ in the coexisting evanescent and propagating eigenmodes becomes very similar, as $\omega$ approaches $\omega_{s}$. This can result in an abnormal interference pattern constituting the frozen mode regime. What exactly happens in the vicinity of a particular stationary point (11) essentially depends on its character and appears to be very different in each of the three cases presented in Fig. 1.

\subsection{Bloch composition of frozen mode}

If all the layers of the periodic layered structure were made of isotropic materials, such as glass or air, each of the Bloch eigenmodes would be doubly degenerate with respect to light polarization. This applies both to propagating and evanescent Bloch modes. In our case, though, each unit cell of the periodic array contains a pair of birefringent layers with misaligned anisotropy axes. As a consequence, the Bloch eigenmodes with different (elliptic) polarization will not be degenerate. At any given frequency, the total number of Bloch eigenmodes with $k \| z$ is four. The only exception are the stationary point frequencies (11), where some of the four eigenmodes cannot be represented in the Bloch form (19). Those cases will be discussed later.

With the exception of stationary points (11), any plane monochromatic wave (16) can be represented as a superposition of four Bloch eigenmodes, propagating and/or evanescent, with different polarizations and wave numbers. But in the setting where the semi-infinite periodic array occupies the half-space $z \geq 0$, the transmitted wave is a superposition of only two of the four Bloch eigenmodes. Indeed, neither the propagating modes with negative group velocity, nor evanescent modes exponentially growing with the distance $z$ from the surface, contribute to $\Psi_{T}(z)$ in this case. Generally, one can distinguish three different possibilities. 
(i) Both Bloch components of the transmitted wave $\Psi_{T}$ are propagating modes

$$
\Psi_{T}(z)=\Psi_{p r 1}(z)+\Psi_{p r 2}(z), \quad z \geq 0
$$

$\Psi_{p r 1}(z)$ and $\Psi_{p r 2}(z)$ are two propagating Bloch modes with different real wave numbers $k_{1}$ and $k_{2}$ and different group velocities $v_{g 1}>0$ and $v_{g 2}>0$. This constitutes the phenomenon of double refraction, provided that $v_{g 1}$ and $v_{g 2}$ are different. The remaining two Bloch modes of the same frequency have negative group velocities and cannot contribute to the transmitted wave $\Psi_{T}$.

(ii) Both Bloch components of $\Psi_{T}$ are evanescent

$$
\Psi_{T}(z)=\Psi_{e v 1}(z)+\Psi_{e v 2}(z), \quad z \geq 0 .
$$

The respective two values of $k$ are complex with positive imaginary parts $k_{1}^{\prime \prime}>0$, $k_{2}^{\prime \prime}>0$. This is the case when the frequency $\omega$ falls into photonic band gap at $\omega>\omega_{g}$ in Fig. 1(a) or at $\omega>\omega_{d}$ in Fig. 1(c). The fact that $k_{1}^{\prime \prime}>0, k_{2}^{\prime \prime}>0$ implies that the wave amplitude decays with the distance $z$ from the surface. In the case (21), the incident wave is totally reflected back to space by the semiinfinite periodic structure.

(iii) One of the Bloch components of the transmitted wave $\Psi_{T}$ is a propagating mode with $v_{g}>0$, while the other is an evanescent mode with $k^{\prime \prime}>0$

$$
\Psi_{T}(z)=\Psi_{p r}(z)+\Psi_{e v}(z), \quad z \geq 0 .
$$

For example, this is the case at $\omega \sim \omega_{0}$ in Fig. 1(b), as well as at $\omega<\omega_{g}$ in Fig. 1(a) and at $\omega<\omega_{d}$ in Fig. 1(c). As the distance $z$ from the surface increases, the evanescent contribution $\Psi_{e v}$ in (22) decays as $\exp \left(-z k^{\prime \prime}\right)$, and the resulting transmitted wave $\Psi_{T}(z)$ turns into a single propagating Bloch mode $\Psi_{p r}$.

Propagating modes with $v_{g}>0$ and evanescent modes with $k^{\prime \prime}>0$ are referred to as forward modes. Only forward modes contribute to the transmitted wave $\Psi_{T}(z)$ in the case of a periodic semi-infinite stack. The propagating modes with $v_{g}<0$ and evanescent modes with $k^{\prime \prime}<0$ are referred to as backward modes. The backward Bloch modes never contribute to the transmitted wave $\Psi_{T}(z)$ inside the periodic semi-infinite stack. This statement is based on the following two assumptions:

- The transmitted wave $\Psi_{T}$ and the reflected wave $\Psi_{R}$ are originated from the plane wave $\Psi_{I}$ incident on the semi-infinite photonic slab from the left.

- The periodic structure occupies the entire half-space and is perfectly periodic at $z>0$.

If either of the above conditions is violated, the electromagnetic field inside the periodic stack can be a superposition of four Bloch eigenmodes with either sign of the group velocity $v_{g}$ of propagating contributions, or either sign of $k^{\prime \prime}$ of evanescent contributions. This would be the case if the periodic array had some kind of structural defects or a finite thickness. At the end of this section we briefly discuss how it would affect the frozen mode regime.

Note also that the assumption that the transmitted wave $\Psi_{T}(z)$ is a superposition of propagating and/or evanescent Bloch eigenmodes may not apply if the frequency $\omega$ exactly coincides with one of the stationary point frequencies (11). For example, at frequency $\omega_{0}$ of stationary inflection point (12), there are no evanescent solutions 
to the Maxwell equations, and the transmitted wave $\Psi_{T}(z)$ is a (non-Bloch) Floquet eigenmode linearly growing with $z\left[35,36\right.$. Similar situation occurs at frequency $\omega_{d}$ of degenerate band edge (13). The term "non-Bloch" means that the respective field distribution does not comply with the relation (19). At the same time, at any general frequency, including the vicinity of any stationary point (11), the transmitted wave $\Psi_{T}(z)$ is a superposition of two forward Bloch eigenmodes, each of which is either propagating, or evanescent.

In all three cases (20-22), the contribution of a particular Bloch eigenmode to the transmitted wave $\Psi_{T}$ depends on the polarization $\Psi_{I}$ of the incident wave. One can always choose some special (elliptic) incident wave polarization, such that only one of the two forward Bloch modes is excited and the transmitted wave $\Psi_{T}$ is a single Bloch eigenmode. In the next subsection we will see that there is no frozen mode regime in the case of a single mode excitation. This fact relates to the very nature of the frozen mode regime in a periodic layered structure [38.

Knowing the Bloch composition of the transmitted wave we can give a semiqualitative description of what happens when the frequency $\omega$ of the incident wave approaches one of the stationary points (11) in Fig. 1 The rigorous mathematical analysis of the respective scattering problem can be found in [35, 38.

3.1.1. Regular photonic band edge We start with the simplest case of a regular photonic band edge. There are two different possibilities in this case, but none of them is associated with the frozen mode regime. The first one relates to the trivial case where none of the layers of the periodic structure displays an in-plane anisotropy or gyrotropy. In this case, all eigenmodes are doubly degenerate with respect to polarization. A detailed description of this case can be found in the extensive literature on optics of stratified media [42, 43. Slightly different scenario occurs if some of the layers are anisotropic or gyrotropic and, as a result, the polarization degeneracy is lifted. Just below the band edge frequency $\omega_{g}$ in Fig. 1(a), the transmitted field $\Psi_{T}(z)$ is a superposition (22) of one propagating and one evanescent Bloch modes. Due to the boundary condition (17), the amplitude of the transmitted wave at $z=0$ is comparable to that of the incident wave. In the case of a generic polarization of the incident light, the amplitudes of the propagating and evanescent Bloch components at $z=0$ are also comparable to each other and to the amplitude of the incident light

$$
\left|\Psi_{p r}(0)\right| \sim\left|\Psi_{e v}(0)\right| \sim\left|\Psi_{I}\right| \text {, at } \omega \leq \omega_{g} .
$$

As the distance $z$ from the surface increases, the evanescent component $\Psi_{e v}(z)$ decays rapidly, while the amplitude of the propagating component remains constant. Eventually, at a certain distance from the slab surface, the transmitted wave $\Psi_{T}(z)$ becomes very close to the propagating mode

$$
\Psi_{T}(z) \approx \Psi_{p r}(z), \text { at } z \gg L, \omega \leq \omega_{g} .
$$

The evanescent component $\Psi_{e v}$ of the transmitted wave does not display any singularity at the band edge frequency $\omega_{g}$. The propagating mode $\Psi_{p r}$ does develop a singularity associated with vanishing group velocity at $\omega \rightarrow \omega_{g}-0$, but its amplitude remains finite and comparable to that of the incident wave. At $\omega>\omega_{g}$, this propagating mode turns into another evanescent mode in (21). The bottom line is that none of the Bloch components of the transmitted wave develops a large amplitude in the vicinity of a regular photonic band edge. There is no frozen mode regime in this trivial case. 
3.1.2. Stationary inflection point A completely different situation develops in the vicinity of a stationary inflection point (12). At $\omega \approx \omega_{0}$, the transmitted wave $\Psi_{T}$ is a superposition (22) of one propagating and one evanescent Bloch component. In contrast to the case of a regular photonic band edge, in the vicinity of $\omega_{0}$ both Bloch contributions to $\Psi_{T}$ develop strong singularity. Specifically, as the frequency $\omega$ approaches $\omega_{0}$, both contributions grow dramatically, while remaining nearly equal and opposite in sign at the slab boundary [35]

$$
\Psi_{p r}(0) \approx-\Psi_{e v}(0) \propto\left|\omega-\omega_{0}\right|^{-1 / 3}, \quad \text { as } \omega \rightarrow \omega_{0} .
$$

Due to the destructive interference (25), the resulting field

$$
\Psi_{T}(0)=\Psi_{p r}(0)+\Psi_{e v}(0)
$$

at the surface at $z=0$ is small enough to satisfy the boundary condition (17). As the distance $z$ from the slab boundary increases, the destructive interference becomes less effective - in part because the evanescent contribution decays exponentially

$$
\Psi_{e v}(z) \approx \Psi_{e v}(0) \exp \left(-z k^{\prime \prime}\right),
$$

while the amplitude of the propagating contribution remains constant and very large. Eventually, the transmitted wave $\Psi_{T}(z)$ reaches its large saturation value corresponding to its propagating component $\Psi_{p r}$, as seen in Fig. 6(a).

Note that the imaginary part $k^{\prime \prime}$ of the evanescent mode wave number in (26) also vanishes in the vicinity of stationary inflection point

$$
k^{\prime \prime} \propto\left|\omega-\omega_{0}\right|^{1 / 3}, \text { as } \omega \rightarrow \omega_{0},
$$

reducing the rate of decay of the evanescent contribution (26). As a consequence, the resulting amplitude $\Psi_{T}(z)$ of the transmitted wave reaches its large saturation value $\Psi_{p r}$ in (25) only at a certain distance $Z$ from the surface

$$
Z \propto 1 / k^{\prime \prime} \propto\left|\omega-\omega_{0}\right|^{-1 / 3} .
$$

This characteristic distance diverges as the frequency approaches its critical value $\omega_{0}$.

If the frequency of the incident wave is exactly equal to the frozen mode frequency $\omega_{0}$, the transmitted wave $\Psi_{T}(z)$ does not reduce to the sum (22) of propagating and evanescent contributions, because at $\omega=\omega_{0}$, there is no evanescent solutions to the Maxwell equations. Instead, $\Psi_{T}(z)$ corresponds to a non-Bloch Floquet eigenmode diverging linearly with $z$ 35.

$$
\Psi_{T}(z)-\Psi_{T}(0) \propto z \Psi_{0}, \text { at } \omega=\omega_{0} .
$$

Such a solution is shown in Fig. 2(c).

3.1.3. Degenerate band edge While the situation with a regular photonic band edge appears trivial, the case of a degenerate band edge (13) proves to be quite different. Just below the degenerate band edge frequency $\omega_{d}$ (inside the transmission band), the transmitted field is a superposition (22) of one propagating and one evanescent components. Above $\omega_{d}$ (inside the band gap), the transmitted wave is a combination (21) of two evanescent components. In this respect, a regular and a degenerate band 
edges are similar to each other. A crucial difference, though, is that in the vicinity of a degenerate band edge, both Bloch contributions to the transmitted wave diverge as $\omega$ approaches $\omega_{d}$, both above and below the band edge frequency. This constitutes the frozen mode regime.

Let us start with the transmission band. As the frequency $\omega$ approaches $\omega_{d}-0$, both Bloch contributions in (22) grow sharply, while remaining nearly equal and opposite in sign at the surface at $z=0,38$.

$$
\Psi_{p r}(0) \approx-\Psi_{e v}(0) \propto\left|\omega_{d}-\omega\right|^{-1 / 4}, \quad \text { as } \omega \rightarrow \omega_{d}-0 .
$$

The destructive interference (30) ensures that the boundary condition (17) can be satisfied, while both Bloch contributions to $\Psi_{T}(z)$ diverge. As the distance $z$ from the slab boundary increases, the evanescent component $\Psi_{e v}(z)$ dies out

$$
\Psi_{e v}(z) \approx \Psi_{e v}(0) \exp \left(-z k^{\prime \prime}\right)
$$

while the propagating component $\Psi_{p r}(z)$ remains constant and very large. Eventually, as the distance $z$ further increases, the transmitted wave $\Psi_{T}(z)$ reaches its large saturation value corresponding to its propagating component $\Psi_{p r}(z)$, as illustrated in Fig. 7 Note that the imaginary part $k^{\prime \prime}$ of the evanescent mode wave number also vanishes in the vicinity of degenerate band edge

$$
k^{\prime \prime} \propto\left|\omega-\omega_{d}\right|^{1 / 4}, \text { as } \omega \rightarrow \omega_{d},
$$

reducing the rate of decay of the evanescent contribution (31). As a consequence, the resulting amplitude $\Psi_{T}(z)$ of the transmitted wave reaches its large saturation value $\Psi_{p r}$ only at a certain distance $Z$ from the surface

$$
Z \propto 1 / k^{\prime \prime} \propto\left|\omega-\omega_{d}\right|^{-1 / 4} .
$$

This characteristic distance increases as the frequency approaches its critical value $\omega_{d}$, as illustrated in Fig. 3(a) and (b).

If the frequency $\omega$ of the incident wave is exactly equal to $\omega_{d}$, the transmitted wave $\Psi_{T}(z)$ does not reduce to the sum of two Bloch contributions. Instead, it corresponds to a non-Bloch Floquet eigenmode linearly diverging with $z$

$$
\Psi_{T}(z)-\Psi_{T}(0) \propto z \Psi_{d}, \text { at } \omega=\omega_{d} .
$$

This situation is shown in Fig. 3(c).

The above behavior appears to be very similar to that of the frozen mode regime at a stationary inflection point, shown in Figs. 2] and 6. Yet, there is a crucial difference between the frozen mode regime at a stationary inflection point and at a degenerate band edge. In the immediate proximity of a degenerate band edge, the Pointing vector $S_{T}$ of the transmitted wave is infinitesimal, in spite of the diverging wave amplitude. In other words, although the energy density $W_{T} \propto\left|\Psi_{T}\right|^{2}$ of the frozen mode diverges as $\omega \rightarrow \omega_{d}-0$, it does not grow fast enough to offset the vanishing group velocity. As a consequence, the photonic crystal becomes totally reflective at $\omega=\omega_{d}$. Of course, the total reflectivity persists at $\omega>\omega_{d}$, where there is no propagating modes at all. By contrast, in the case (29) of a stationary inflection point, the respective Pointing vector $S_{T}$ is finite and can be even close to that of the incident wave, implying low 
reflectivity and nearly total conversion of the incident wave energy into the frozen mode.

The character of frozen mode regime is different when we approach the degenerate band edge frequency from the band gap. In such a case, the transmitted field $\Psi_{T}(z)$ is a superposition (21) of two evanescent components. As the frequency $\omega$ approaches $\omega_{d}$, both evanescent contributions grow sharply, while remaining nearly equal and opposite in sign at the photonic crystal boundary

$$
\Psi_{e v 1}(0) \approx-\Psi_{e v 2}(0) \propto\left|\omega_{d}-\omega\right|^{-1 / 4}, \quad \text { as } \omega \rightarrow \omega_{d}+0 .
$$

Again, the destructive interference (35) ensures that the boundary condition (17) can be satisfied, while both evanescent contributions to $\Psi_{T}(z)$ diverge in accordance with (35). As the distance $z$ from the slab boundary increases, the destructive interference of these two evanescent components is lifted and the resulting field amplitude increases sharply, as seen in Fig. 8(a). But eventually, as the distance $z$ further increases, the transmitted wave $\Psi_{T}(z)$ completely decays, because both Bloch contributions to $\Psi_{T}(z)$ are evanescent. The latter constitutes the major difference between the frozen mode regime above and below the DBE frequency $\omega_{d}$. The rate of the amplitude decay, as well as the position of the maximum of the transmitted wave amplitude in Figs. 3( $(\mathrm{d}-\mathrm{f})$ and $8(\mathrm{a})$, are characterized by the distance $Z$ in (33).

3.1.4. Physical reason for the growing wave amplitude If the frequency $\omega$ is close, but not equal, to that of a stationary point (11) of the dispersion relation, the wave $\Psi_{T}(z)$ transmitted to the semi-infinite periodic layered structure is a superposition of two forward Bloch modes $\Psi_{1}(z)$ and $\Psi_{2}(z)$

$$
\Psi_{T}(z)=\Psi_{1}(z)+\Psi_{2}(z), z \geq 0 .
$$

The two Bloch modes in (36) can be one propagating and one evanescent, as in (22), or they can be both evanescent, as in (21). In the vicinity of frozen mode regime, as the frequency approaches its critical value of $\omega_{0}$ or $\omega_{d}$, the four-dimensional vectors (16) corresponding to each of the two Bloch eigenmodes on the right-hand side of (36) become nearly parallel to each other

$$
\Psi_{1}(z) \approx \alpha \Psi_{2}(z), \quad \text { as } \omega \rightarrow \omega_{s},
$$

where $\alpha$ is a scalar, and $\omega_{s}$ is the frozen mode frequency $\omega_{0}$ or $\omega_{d}$. The asymptotic relation (37) reflects a basic property of the transfer matrix of the periodic layered structure at the frequency of either stationary point $\omega_{0}$ or $\omega_{d}$ (see [36-38]).

Let us show how the property (37) of the Bloch eigenmodes in the vicinity of $\omega_{0}$ or $\omega_{d}$ can lead to the frozen mode regime. Indeed, at the photonic crystal boundary, the sum (36) of two nearly parallel column vectors $\Psi_{1}(z)$ and $\Psi_{2}(z)$ must match the boundary conditions (17) with the incident and reflected waves $\Psi_{I}(0)$ and $\Psi_{R}(0)$. If the incident wave polarization is general, we have no reason to expect the columnvector

$$
\Psi(0)=\Psi_{I}(0)+\Psi_{R}(0)
$$

at the photonic crystal interface to be nearly parallel to $\Psi_{1}(0)$ and $\Psi_{2}(0)$. But on the other hand, the boundary condition (17) says that

$$
\Psi(0)=\Psi_{1}(0)+\Psi_{2}(0)
$$


Obviously, the only situation where $\Psi(0)$ in (38) and (39) is a general four-dimensional vector, while $\Psi_{1}(0)$ and $\Psi_{2}(0)$ on the right-hand side of (39) are nearly parallel to each other is when

$$
\Psi_{1}(0) \approx-\Psi_{2}(0), \quad\left|\Psi_{1}(0)\right| \approx\left|\Psi_{2}(0)\right| \gg|\Psi(0)| .
$$

The relation (40) explains how the huge diverging amplitude of the frozen mode inside the photonic crystal can be reconciled with the boundary conditions (17).

There is one exception, though. As we already stated in (37), in the vicinity of the frozen mode frequency, the two Bloch components $\Psi_{1}(z)$ and $\Psi_{2}(z)$ of the transmitted wave $\Psi_{T}(z)$ are nearly parallel to each other. For this reason, if the polarization of the incident wave $\Psi_{I}$ is such that $\Psi(0)$ in (39) is nearly parallel to one of the Bloch eigenmodes $\Psi_{1}(0)$ or $\Psi_{2}(0)$, it is also nearly parallel to the other one. So, all three column vectors $\Psi_{1}(0), \Psi_{2}(0)$, and $\Psi(0)$ are now parallel to each other. In this, and only this case, the amplitude of the transmitted wave $\Psi_{T}(z)$ will be comparable to that of the incident wave. There is no frozen mode regime for the respective vanishingly small range of the incident wave polarization. A particular case of the above situation is the regime of a single mode excitation, where only one of the two Bloch components $\Psi_{1}(z)$ or $\Psi_{2}(z)$ in (36) contributes to the transmitted wave $\Psi_{T}(z)$ 38. Indeed, if the transmitted wave is a single Bloch mode (either $\Psi_{1}(z)$, or $\Psi_{2}(z)$ ), the condition (40) cannot be satisfied.

Finally, let us reiterate that in the limiting cases of $\omega=\omega_{0}$ or $\omega=\omega_{d}$, the transmitted wave $\Psi_{T}(z)$ corresponds to the non-Bloch Floquet eigenmode (29) or (34), respectively. Either of them linearly diverges with $z$. The only exception is when the incident wave has the unique (elliptic) polarization, for which the transmitted wave $\Psi_{T}(z)$ is a propagating Bloch eigenmode with zero group velocity and a limited amplitude, comparable to that of the incident wave. Incident wave with any other polarization will generate the frozen mode inside the periodic medium.

\section{Summary}

Although the existence of slow electromagnetic modes in photonic crystals is quite obvious, the next question is whether and how such modes can be excited by incident light. We have shown that the frozen mode regime provides unique advantages in this respect. Generally, the possibility of the frozen mode regime is determined by the character of the Bloch dispersion relation, rather than the specific physical realization of the periodic structure supporting such a dispersion relation. As soon as the Bloch dispersion relation displays the proper singularity such as a stationary inflection point (12) or a degenerate band edge (13), we have every reason to expect the occurrence of the frozen mode regime at the respective frequency. In other words, the possibility of the frozen mode regime is determined by the spectral properties of the periodic structure, rather than by specific physical nature of the linear excitations.

If a periodic structure is relatively simple - for instance, a stratified medium with one dimensional periodicity - its frequency spectrum may prove to be too simple to support the proper spectral singularity and the frozen mode regime. All our numerical examples relate to $1 D$ photonic crystals, where the existence of the frozen mode regime requires the presence of misaligned birefringent layers (see the details in [38). The more complex the periodic structure is, the more likely it is capable of supporting such a phenomenon. In photonic crystals with $3 D$ periodicity, there are no fundamental 
restrictions on the existence of the frozen mode regime [46]. The same is true for the cases of modulated waveguides and periodic arrays of coupled resonators.

Another important question is how robust the frozen mode regime is. For instance, what happens if we introduce absorption or structural imperfections. Of course, all these factors suppress the frozen mode amplitude, but not as much as in the case of common Fabry-Perot or transmission band edge resonances, where the entire photonic structure works as a resonator. In addition, since in the vicinity of an inflection point the dispersion term $v_{g}^{\prime}(k)=\omega^{\prime \prime}(k)$ vanishes, one can achieve widebandwidth and dispersion-free propagation of light [27.

Finally, it is possible to combine the frozen mode regime and a common slow wave resonance, in which case we have the phenomenon called the giant transmission resonance [39, 40]. The Q-factor associated with such a resonance can be by two orders of magnitude higher, compared to that of the regular Fabry-Perot resonance in the same or similar periodic structure.

Acknowledgment and Disclaimer: Effort of A. Figotin and I. Vitebskiy is sponsored by the Air Force Office of Scientific Research, Air Force Materials Command, USAF, under grant number FA9550-04-1-0359.

[1] L. Brillouin. Wave Propagation and Group Velocity. (Academic, New York, 1960).

[2] L. D. Landau, E. M. Lifshitz, L. P. Pitaevskii. Electrodynamics of continuous media. (Pergamon, N.Y. 1984).

[3] S. E. Harris. Electromagnetically induced transparency. Physics Today 50, \# 7, 36 (1997).

[4] L. Hau, S. Harris, Z. Dutton, C. Behroozi. Light speed reduction to 17 metres per second in an ultracold atomic gas. Nature, 397, $594-598$, (1999).

[5] M. Kash, V. Sautenkov, Al. Zibrov, L. Hollberg, G. Welch, M. Lukin, Yu. Rostovtsev, E. Fry, and M. Scully. Ultraslow Group Velocity and Enhanced Nonlinear Optical Effects in a Coherently Driven Hot Atomic Gas. Phys. Rev. Lett. 82, \#26, 5229 - 5232 (1999)

[6] D. Budker, D. F. Kimball, S. M. Rochester, and V. V. Yashchuk. Nonlinear Magneto-optics and Reduced Group Velocity of Light in Atomic Vapor with Slow Ground State Relaxation. Phys. Rev. Lett. 83, \# 9, 1767 (1999).

[7] M. Lukin and A. Imamoglu. Controlling photons using electromagnetically induced transparency. Nature, 413, 273 - 276, (2001).

[8] D. F. Phillips, A. Fleischhauer, A. Mair, and R. L. Walsworth, M. D. Lukin. Storage of Light in Atomic Vapor. Phys. Rev. Lett. 86, \# 5, 783 - 786, (2001).

[9] A.V. Turukhin, V. S. Sudarshanam, M. S. Shahriar, J. A. Musser, B. S. Ham, and P. R. Hemmer. Observation of Ultraslow and Stored Light Pulses in a Solid. Phys. Rev. Lett. 88, \# 2, 023602 (2002).

[10] G. Agarwal and T. Dey. Non-electromagnetically induced transparency mechanisms for slow light. Laser \& Photon. Rev., 1-14 (2009)

[11] J. Joannopoulos, R. Meade, and J. Winn. Photonic Crystals. (Princeton University Press, 1995).

[12] S. Johnson and J. Joannopoulos. Photonic Crystals. (Kluwer Academic Publisher, 1995).

[13] K. Sakoda. Optical Properties of Photonic Crystals. (Springer, 2001).

[14] A. Yariv and Pochi Yeh. Optical Waves in Crystals. ("A Wiley-Interscience publication", 1984).

[15] J. Heebner and R. Boyd. Slow and stopped light. Slow and fast light in resonator-coupled waveguides. Journal of modern optics, 49, \#14/15, 2629 (2002).

[16] J. Heebner and R. Boyd. Slow light, induced dispersion, enhanced nonlinearity, and optical solitons in a resonator-array waveguide. Phys. Rev. E65, 036619 (2002).

[17] A. Melloni, F. Morichetti, M. Maritelli. Linear and nonlinear pulse propagation in coupled resonator slow-wave optical structures. Optical and Quantum Electronics 35, 365 (2003)

[18] J. Poon, J. Scheuer, Y. Xu, and A. Yariv. Designing coupled-resonator optical waveguide delay lines. J. Opt. Soc. Am. B, Vol. 21, No. 9 (2004).

[19] J. Scheuer, G. Paloczi, J. Poon, and A. Yariv. Toward the Slowing and Storage of Light. OPN, $16,36(2005)$.

[20] J. B. Khurgin. Optical buffers based on slow light in electromagnetically induced transparent media and coupled resonator structures: comparative analysis. J. Opt. Soc. Am. B 22, \#5, $1062(2005)$. 
[21] J. B. Khurgin. Expanding the bandwidth of slow-light photonic devices based on coupled resonators. Optic Letters, 30, \# 5, 513 (2005).

[22] M. Yanik and S. Fan. Stopping Light All Optically. Phys. Rev. Lett. 92, \# 8, 083901, (2004).

[23] M. Terrel, M. Digonnet, and S. Fan. Performance comparison of slow-light coupled-resonator optical gyroscopes. Laser \& Photon. Rev., 1-14 (2009).

[24] M. Notomi,1 K. Yamada,2 A. Shinya,1 J. Takahashi,2 C. Takahashi,2 and I. Yokohama. Extremely Large Group-Velocity Dispersion of Line-Defect Waveguides in Photonic Crystal Slabs. Phys. Rev. Lett. 87, \#25, 253902 (2001).

[25] D. Mori and T. Baba. Wideband and low dispersion slow light by chirped photonic crystal coupled waveguide. OPTICS EXPRESS, 13, 9398(2005)

[26] T. Baba, T. Kawasaki, H. Sasaki, J. Adachi, and D. Mori. Large delay-bandwidth product and tuning of slow light pulse in photonic crystal coupled waveguide. OPTICS EXPRESS, 16, 9245(2008)

[27] T. Baba. Slow light in photonic crystals (Review). Nature (photonics), 2, 465(2008)

[28] T. Baba and D. Mori. Slow light engineering in photonic crystals. J. Phys. D: Appl. Phys. 40, 2659(2007)

[29] S.-C. Huang, M. Kato, E. Kuramochi, C.-P. Lee, and M. Notomi. Time-domain and spectral-domain investigation of inflection-point slow-light modes in photonic crystal coupled waveguides. OPTICS EXPRESS, 15, 3543(2007)

[30] Jing Ma and Chun Jiang. Flatband Slow Light in Asymmetric Line-Defect Photonic Crystal Waveguide Featuring Low Group Velocity and Dispersion. IEEE J. Quant. Electr., 44, 763(2008).

[31] J. Li, Th. White, L. O'Faolain1, A. Gomez-Iglesias, and T. Krauss. Systematic design of flat band slow light in photonic crystal waveguides. OPTICS EXPRESS Vol. 166227 (2008)

[32] M. Scalora, R. J. Flynn, S. B. Reinhardt, R. L. Fork, M. J. Bloemer, M. D. Tocci, C. M. Bowden, H. S. Ledbetter, J. M. Bendickson, J. P. Dowling, R. P. Leavitt. Ultrashort pulse propagation at the photonic band edge: Large tunable group delay with minimal distortion and loss. Phys. Rev. E54, \#2, R1078 (1996).

[33] M. Bloemer, K. Myneni, M. Centini, M. Scalora, and G. D'Aguanno. Transit time of optical pulses propagating through a finite length medium. Phys. Rev. E65, 056615 (2002).

[34] M. Soljacic, S. Johnson, S. Fan, M. Ibanescu, E. Ippen, and J. D. Joannopoulos. Photoniccrystal slow-light enhancement of nonlinear phase sensitivity. J. Opt. Soc. Am. B., 19, \#9, 2052 (2002).

[35] A. Figotin, and I. Vitebskiy. Electromagnetic unidirectionality in magnetic photonic crystals. Phys. Rev. B67, 165210 (2003).

[36] A. Figotin, and I. Vitebskiy. Oblique frozen modes in layered media. Phys. Rev. E68, 036609 (2003).

[37] J. Ballato, A. Ballato, A. Figotin, and I. Vitebskiy. Frozen light in periodic stacks of anisotropic layers. Phys. Rev. E71, (2005).

[38] A. Figotin and I. Vitebskiy. Frozen light in photonic crystals with degenerate band edge. Phys. Rev. E74, 066613 (2006)

[39] A.Figotin and I.Vitebskiy. Gigantic transmission band-edge resonance in periodic stacks of anisotropic layers. Phys. Rev. E72, 036619, (2005).

[40] A. Figotin and I. Vitebskiy. Slow-wave resonance in periodic stacks of anisotropic layers. Phys. Rev. A76, 053839 (2007).

[41] A.Figotin and I.Vitebskiy. Electromagnetic unidirectionality and frozen modes in magnetic photonic crystals. JMMM, 300, 117 (2006).

[42] Pochi Yeh. "Optical Waves in Layered Media", (Wiley, New York, 1988).

[43] Weng Cho Chew. "Waves and Fields in Inhomogeneous Media", (Van Nostrand Reinhold, New York, 1990).

[44] M. Notomi. Theory of light propagation in strongly modulated photonic crystals: Refractionlike behavior in the vicinity of the photonic band gap. Phys. Rev. B62, 10696 (2000)

[45] A. Figotin, and I. Vitebsky. Nonreciprocal magnetic photonic crystals. Phys. Rev. E63, 066609 (2001).

[46] H. Noh, M. Scharrer, M. Anderson, R. Chang, and Hui Cao. Photoluminescence modification by a high-order photonic band with abnormal dispersion in $\mathrm{ZnO}$ inverse opal. Phys. Rev. B77, 115136 (2008). 


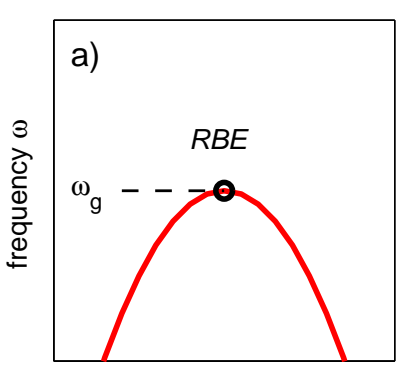

wave number $k$

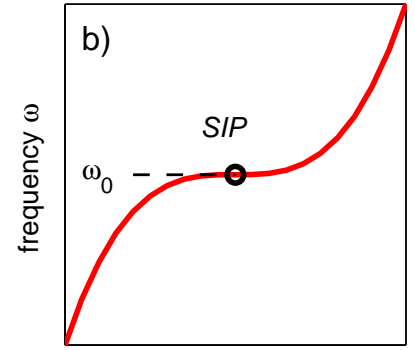

wave number $k$

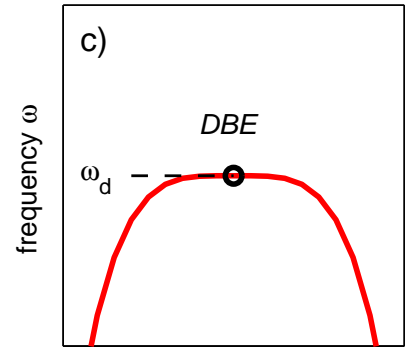

wave number $k$

Figure 1. (Color online). Schematic examples of dispersion relations displaying different stationary points: (a) a regular band edge (RBE), (b) a stationary inflection point (SIP), (c) a degenerate band edge (DBE). 

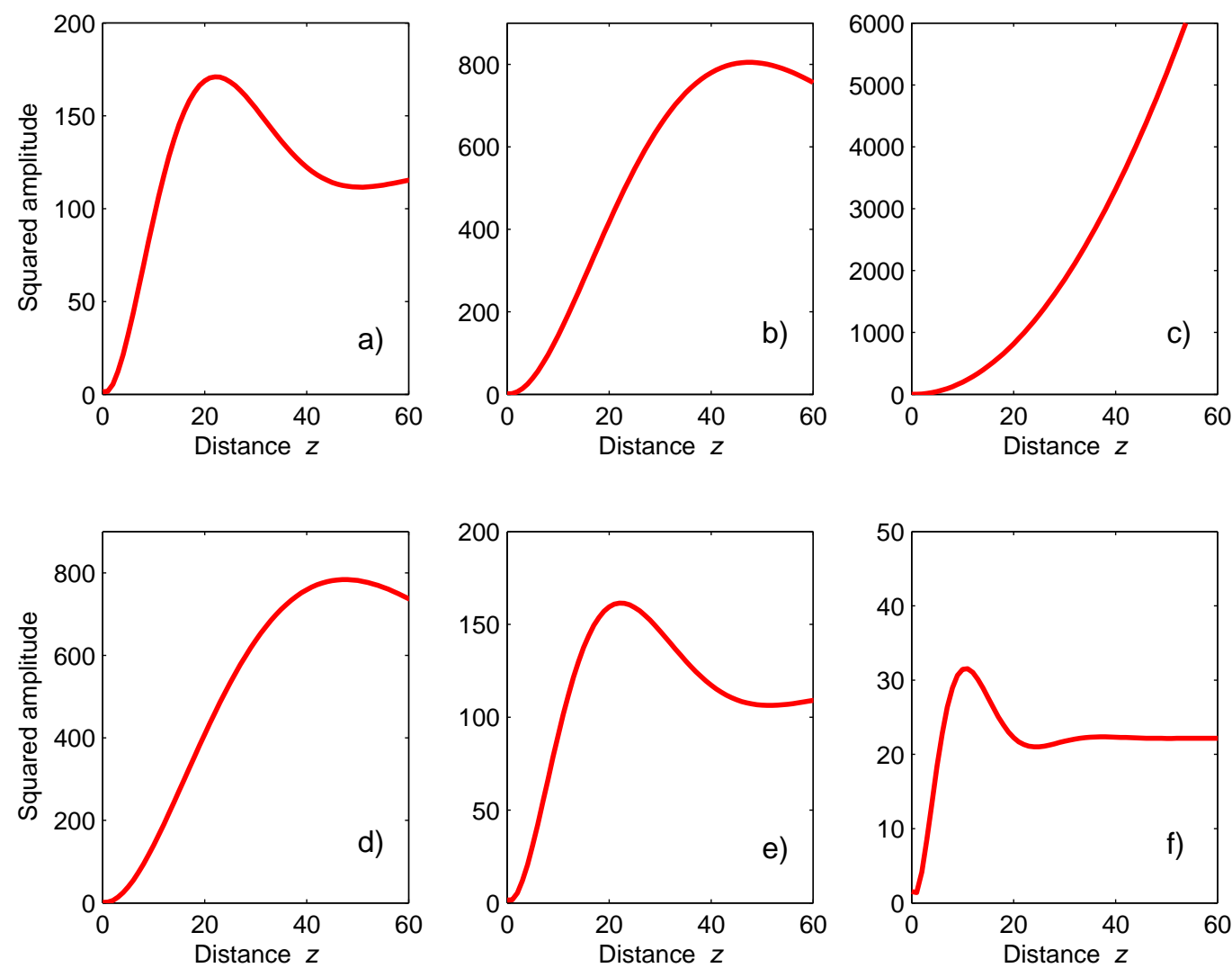

Figure 2. (Color online). Smoothed profile of the frozen mode at six different frequencies in the vicinity of stationary inflection point: (a) $\omega=\omega_{0}-10^{-4} c / L$, (b) $\omega=\omega_{0}-10^{-5} c / L$, (c) $\omega=\omega_{0}$, (d) $\omega=\omega_{0}+10^{-5} c / L$, (e) $\omega=\omega_{0}+10^{-4} c / L$, (f) $\omega=\omega_{0}+10^{-3} c / L$. In all cases, the incident wave has the same polarization and unity amplitude. The distance $z$ from the surface of semi-infinite photonic crystal is expressed in units of $L$. 

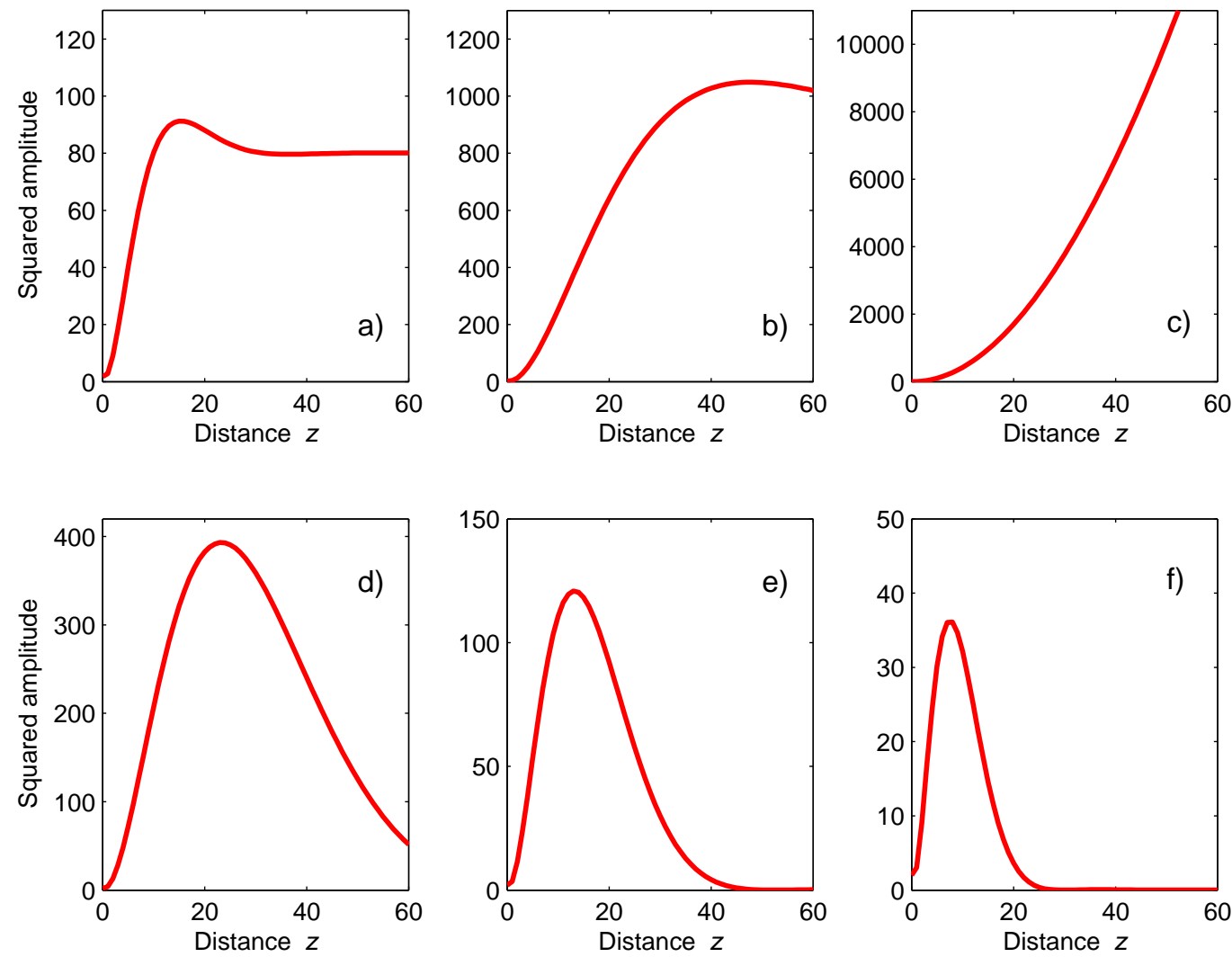

Figure 3. (Color online). Smoothed profile of the frozen mode at six different frequencies in the vicinity of degenerate band edge: (a) $\omega=\omega_{d}-10^{-4} c / L$, (b) $\omega=\omega_{d}-10^{-6} c / L$, (c) $\omega=\omega_{d}$, (d) $\omega=\omega_{d}+10^{-6} c / L$, (e) $\omega=\omega_{d}+10^{-5} c / L$, (f) $\omega=\omega_{d}+10^{-4} c / L$. In the transmission band (at $\omega<\omega_{d}$ ), the asymptotic field value diverges as $\omega \rightarrow \omega_{d}$. By contrast, in the band gap (at $\omega>\omega_{d}$ ), the asymptotic field value is zero. The amplitude of the incident wave at $z<0$ is unity. The distance $z$ from the surface is expressed in units of $L$. 

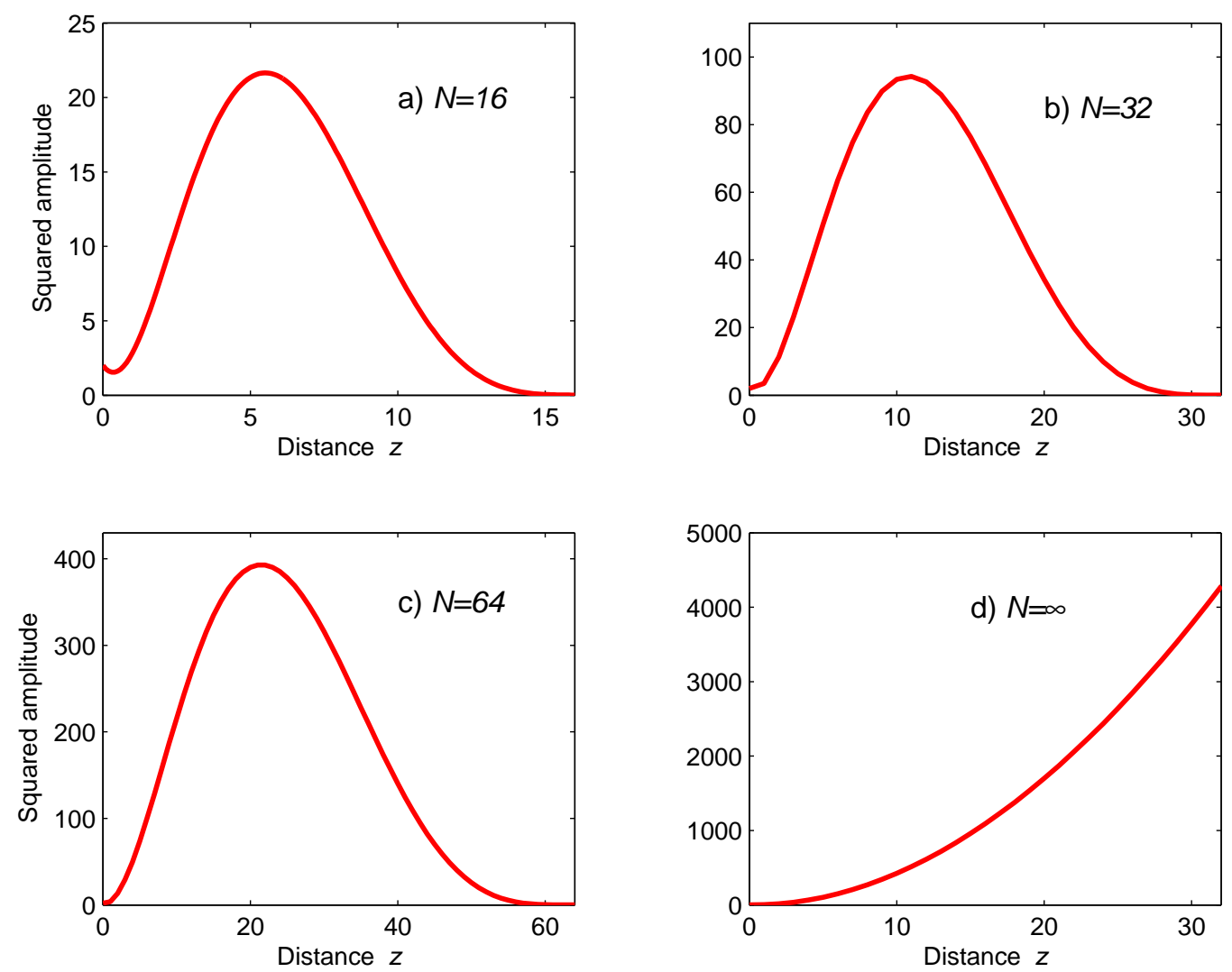

Figure 4. (Color online). Smoothed profile of the frozen mode in periodic layered structures composed of different number $N$ of unit cells $L$. The frequency is equal to that of the degenerate band edge. The initial rate of growth of the frozen mode amplitude is virtually independent of $N$. The limiting case (d) of the semi-infinite structure is also shown in Fig. 3. (c). In all cases, the incident wave has the same polarization and unity amplitude. The distance $z$ from the surface is expressed in units of $L$. 


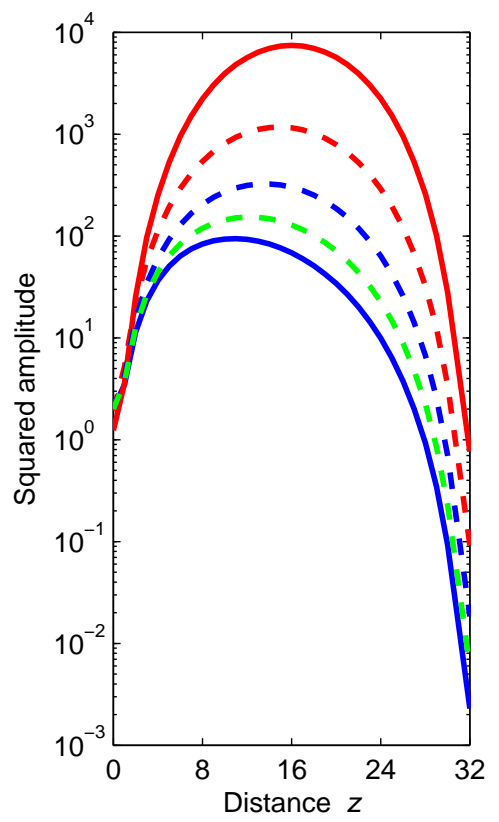

Figure 5. (Color online) Steady-state field distribution in a lossless periodic finite slab with $N=32$ at a series of equidistant frequencies lying between the DBE frequency $\omega_{d}$ and the frequency $\omega_{r}$ of the giant slow wave resonance. Observe that the field amplitude increases dramatically as the frequency changes from that of the DBE (the lowest solid blue curve) to that of the giant slow wave resonance (the upper solid red curve). The lowest solid curve is identical to that in Fig. 4(b). 

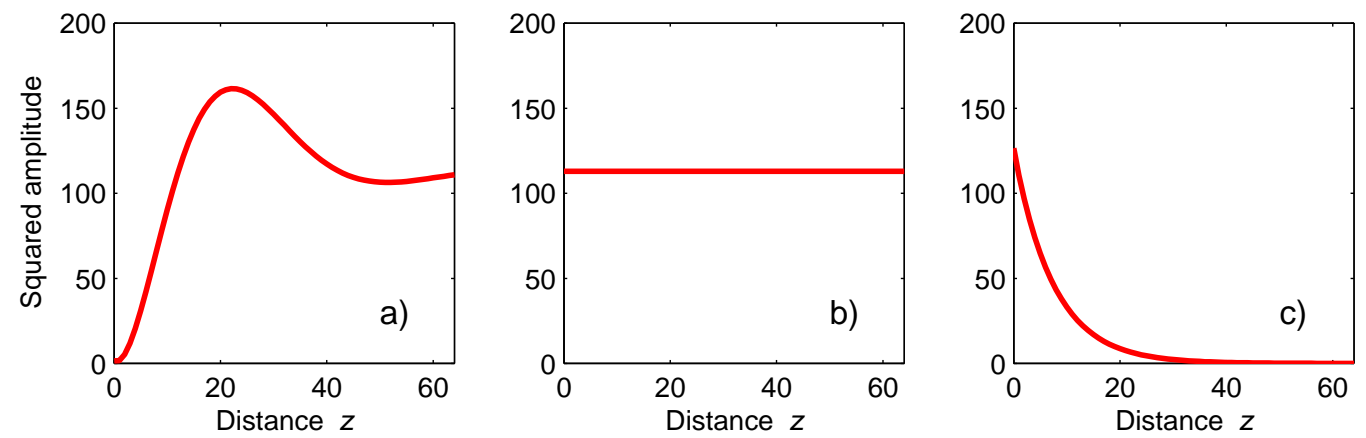

Figure 6. (Color online). Destructive interference of the propagating and evanescent components of the transmitted wave inside semi-infinite photonic crystal. The frequency is close but not equal to that of stationary inflection point. (a) The squared modulus of the resulting transmitted field - its amplitude at $z=0$ is small enough to satisfy the boundary conditions; (b) the squared modulus of the propagating contribution, which is independent of $z ;(\mathrm{c})$ the squared modulus of the evanescent contribution, which decays with the distance $z$. The amplitude of the incident wave is unity. The distance $z$ from the surface is expressed in units of $L$.
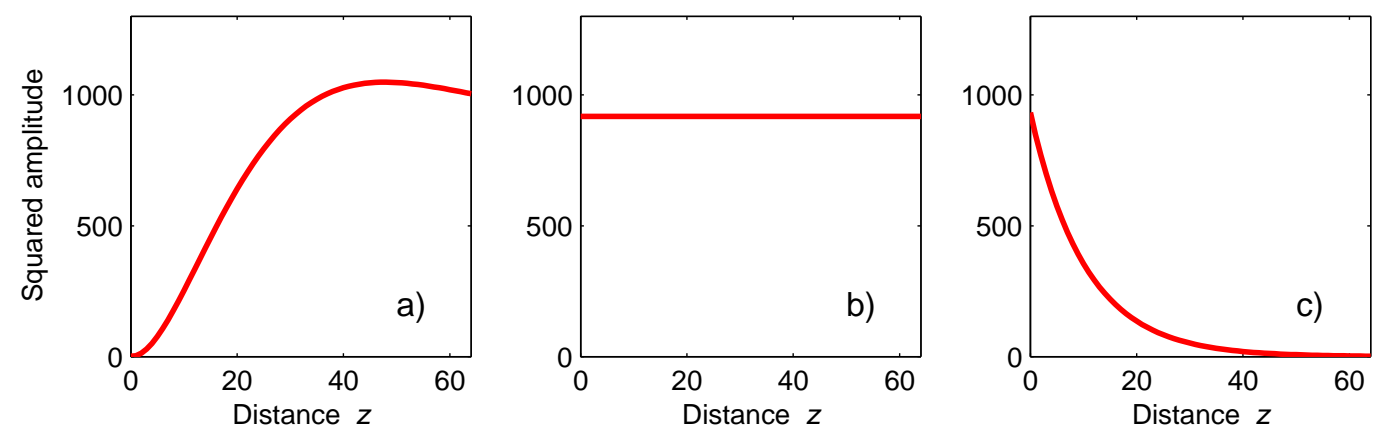

Figure 7. (Color online). Destructive interference of the two Bloch components of the transmitted wave inside semi-infinite photonic crystal. The frequency is $\omega=\omega_{d}-10^{-4} c / L$, which is slightly below the degenerate band edge in Fig. 11(c). (a) The squared modulus of the resulting transmitted field - its amplitude at $z=0$ is small enough to satisfy the boundary conditions (17); (b) the squared modulus of the propagating contribution, which is independent of $z$; (c) the squared modulus of the evanescent contribution, which decays with the distance $z$. The amplitude of the incident wave is unity. 

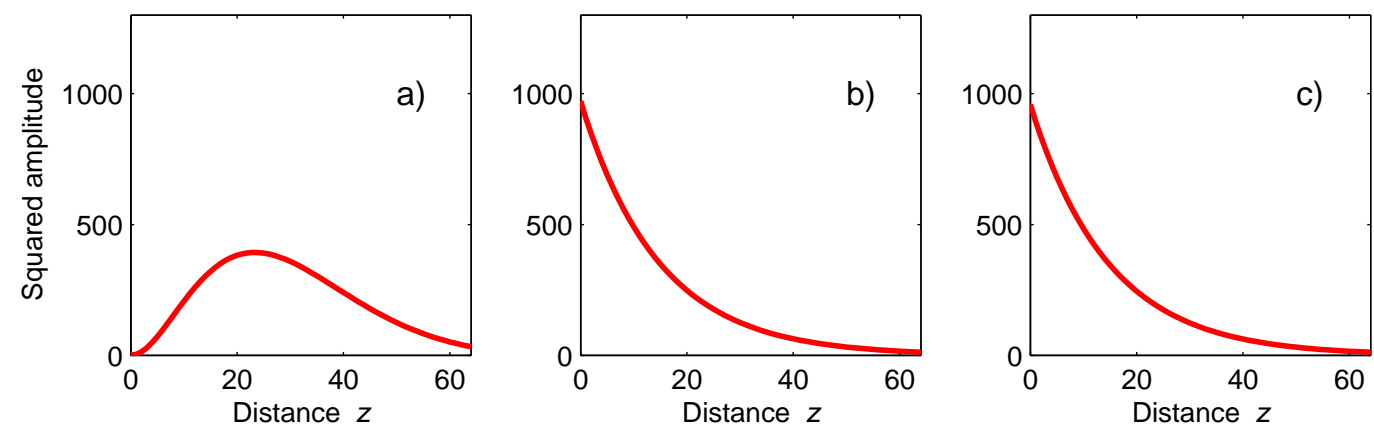

Figure 8. (Color online). Destructive interference of the two Bloch components of the transmitted wave inside semi-infinite photonic crystal. The frequency is $\omega=\omega_{d}+10^{-5} c / L$, which is just above the degenerate band edge. (a) The squared modulus of the resulting transmitted field - its amplitude at $z=0$ is small enough to satisfy the boundary conditions (17); (b) and (c) the squared moduli of the two evanescent contributions; both decay with the distance $z$. The amplitude of the incident wave is unity. 